\title{
Healing and repair after myocardial infarction: the forgotten but resurgent basophil
}

\author{
Sumanth D. Prabhu \\ Department of Medicine, Division of Cardiovascular Disease and Comprehensive Cardiovascular Center, University of Alabama at Birmingham, Birmingham, Alabama, USA.
}

\begin{abstract}
The biphasic wound-healing response in the heart after myocardial infarction involves an initial inflammatory phase followed by a more prolonged period of inflammation resolution, tissue repair, and scar formation. Infiltrating proinflammatory Ly6C ${ }^{\text {hi }}$ monocytes and monocytederived macrophages are key drivers of the inflammatory phase and are also the source of the locally generated reparative macrophages that promote inflammation resolution. In this issue of the $J C I$, Sicklinger et al. from the Leuschner laboratory uncover a salutary role for cardiac basophils in this process. The authors demonstrated that basophils promote healing and proper scar formation and also limit late cardiac remodeling by augmenting reparative macrophages in the infarcted heart, in part via basophil-derived enhancement of cardiac IL-4 and IL-13 levels. These findings underscore the potentially disproportionate (relative to cell numbers) yet essential biological effects of immune cells of low abundance on cardiac repair and remodeling, related in part to amplification of downstream macrophage responses via secreted cytokines.
\end{abstract}

\section{Inflammation and repair after myocardial infarction}

The magnitude of ischemic injury and a well-orchestrated inflammatory and wound-healing response are key determinants of the fidelity of cardiac repair after myocardial infarction (MI); larger infarctions and excessive and/or prolonged inflammatory responses increase the risk for developing late adverse ventricular remodeling and heart failure (HF) (1). The extent of injury is mitigated, and long-term outcomes improved, by early coronary reperfusion for myocardial salvage, which is the standard of care and routinely applied in clinical practice. In contrast, translating therapies to favorably modulate the accompanying sterile inflammatory response has proved much more difficult, in large part because of an incomplete understanding of the various facets of the immune responses that drive tissue inflammation and its subsequent resolution.

The wound-healing response after MI is biphasic, with initial intense inflammation, tissue digestion, and dead cell clearance, subsequently followed by a resolution phase that leads to scar formation (1-5). In mouse models of MI, the inflammatory phase is dominated early by infiltrating neutrophils and subsequently by Ly6C ${ }^{\text {hi }}$ CCR $2{ }^{+}$CX3CR $1^{\text {lo }}$ monocytes and proinflammatory macrophages (post-MI days 0-4; refs. 2, 4-7). During the resolution phase (days 4-14), reparative Ly6C ${ }^{\text {lo }}$ macrophages are generated locally and proliferate in the heart, a process that requires the nuclear hormone receptor Nr4a1 (2). Hence, potential fruitful avenues for modulating the post-MI immune response include both temporally appro-

\section{Delated Article: https://doi.org/10.1172/JCl136778}

Conflict of interest: The author has declared that no conflict of interest exists.

priate suppression of proinflammatory monocytes and macrophages, as well as enhancement of the transition to reparative macrophage phenotypes to usher in resolution. However, regarding the latter, although the macrophage phenotypic switch is facilitated by efferocytosis of apoptotic cardiomyocytes and neutrophils $(1,8,9)$, the specific microenvironmental cues after MI that are responsible remain incompletely defined.

Importantly, several other immune cell types including DCs (10), CD4 ${ }^{+} \mathrm{T}$ cells (11), Tregs (12), and invariant NK T (iNKT) cells (13) promote wound healing and ameliorate adverse cardiac remodeling after MI, in part by favorably influencing monocyte trafficking and/or macrophage differentiation and facilitating antiinflammatory cytokine expression and collagen matrix formation in the heart. In contrast, $B$ cells aggravate tissue injury and adverse remodeling by enhancing proinflammatory Ly6 $\mathrm{C}^{\text {hi }}$ monocyte mobilization to the acutely infarcted heart (14). Hence, interactive responses between different immune cell types and monocytes and macrophages are of particular importance in determining the sufficiency of the postMI healing response.

\section{Another understanding of basophils after MI}

In this issue of the JCI, Sicklinger et al. (15) report a different role for basophils (Figure 1). The rare granulocyte population classically associated with allergic responses and immunity to parasites is involved in the regulation of post-MI healing and the resolution response. The authors performed a series of elegant studies in mice using complementary techniques and models and reported five main findings. First, basophils infiltrated the nonreperfused infarcted heart, peaking primarily during the resolution phase. The increase in basophils was accompanied by augmented myocardial expression of mediators of basophil recruitment (e.g., IL-33). Second, 

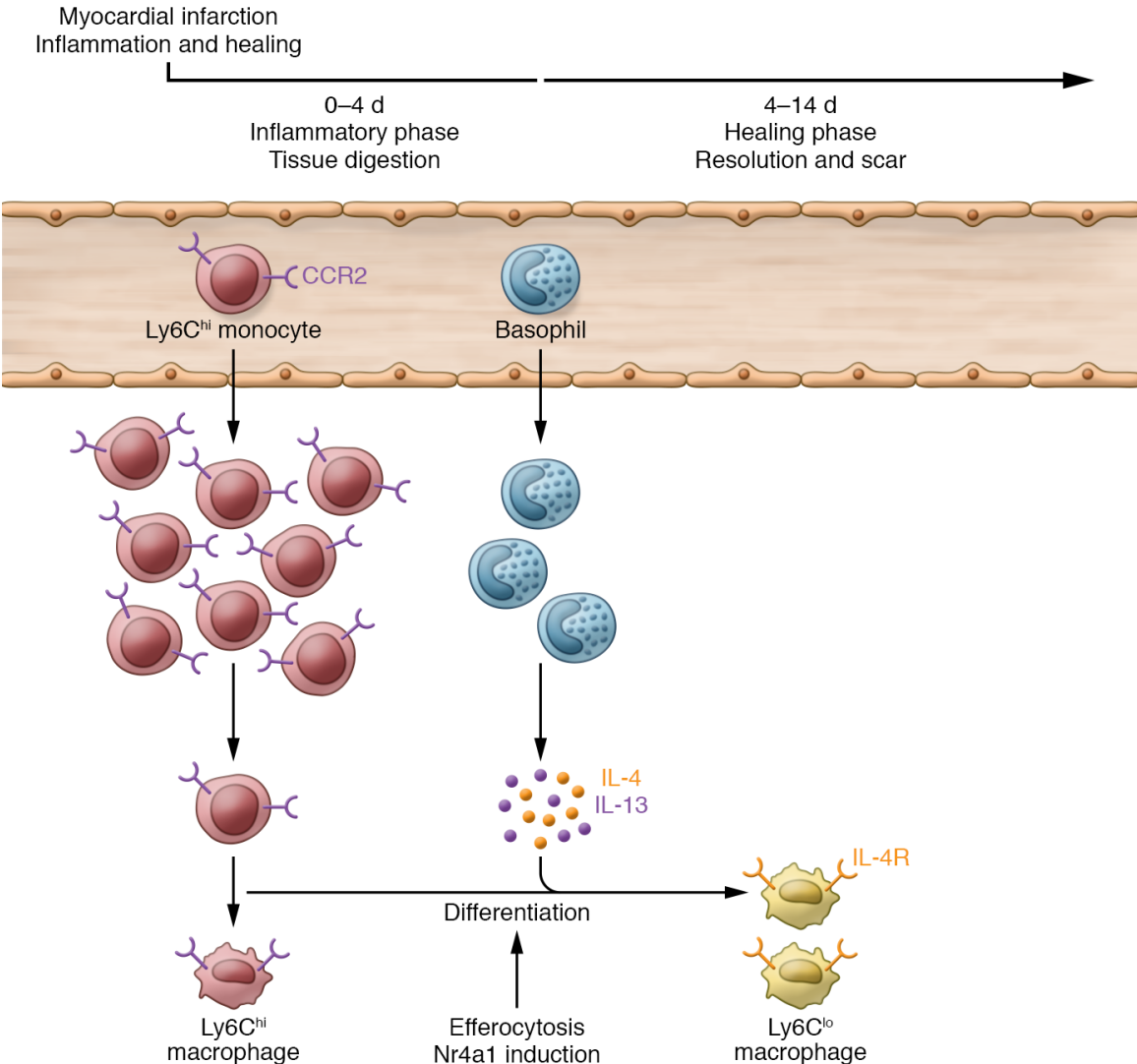

antibody-mediated or genetic depletion of basophils prior to MI resulted in thinner scars and greater adverse remodeling and dysfunction. The lack of basophils altered cardiac (but not systemic) proinflammatory Ly6C $C^{\text {hi }}$ monocytes and decreased reparative Ly6C $\mathrm{C}^{\text {lo }}$ macrophages. Notably, these alterations were reversed upon adoptive transfer of splenic basophils. Third, basophil-specific IL-4/IL-13 deficiency produced a similar phenotype. Importantly, basophils contributed to IL-4/IL-13 production in the infarcted heart. Fourth, inducing basophils to produce IL-4 after acute MI improved post-MI remodeling. Last, the authors examined humans following ST-elevation MI (STEMI). Blood basophil counts increased over a four-day period after acute STEMI. Lower basophil counts correlated, after adjustment for several covariates, with increased scar size as measured by cardiac MRI one year later.

Collectively, these studies have uncovered another aspect of basophil biology and have identified this innate immune cell as a central player in the post-MI healing and resolution response. Specifically, these cells promote healing, resolution, and proper scar formation and thereby lim- it late adverse cardiac remodeling, at least in part via IL-4-mediated effects on the more abundant populations of monocytes and macrophages in the infarcted heart.

\section{Conceptual and translational implications}

These pivotal results highlight important conceptual and translational caveats regarding therapeutic targeting of the immune response after MI and in post-MI HF. Monocytes and macrophages are the most abundant immune cell type in the infarcted heart and thus the most prominent as innate immune effector cells (1, 5); hence, the focus on their biological role and tissue responses and their potential as direct therapeutic targets is readily understandable. However, Sicklinger et al. (15) have demonstrated that low-abundance cell populations (or subpopulations) in the heart, such as basophils, may have disproportionate effects (relative to cell number) on cardiac repair and remodeling. Secreted cytokines and mediators referable to these populations may amplify cellular responses (16) that will subsequently affect the predominant monocyte/macrophage phenotype. Hence, rarer populations of
Figure 1. Schematic of the inflammatory and healing response after MI. In mice, the wound repair process after $\mathrm{MI}$ involves an early inflammatory phase and an inflammation resolution phase. The inflammatory phase is primarily dominated by infiltrating proinflammatory Ly6C ${ }^{\text {hi }}$ monocytes and macrophages. The more prolonged healing phase leads to scar formation and is characterized by reparative $\mathrm{Ly}_{6 \mathrm{C}}{ }^{\mathrm{lo}}$ macrophages generated locally in the heart. Reparative macrophage differentiation is facilitated by efferocytosis and requires the nuclear hormone receptor $\mathrm{Nr} 4 \mathrm{a} 1$. In this issue of the $J \mathrm{Cl}$, Sicklinger et al. (15) report a central role for basophils in the healing process, in large part related to their secretion of IL-4 and IL-13, which in turn promotes reparative macrophage differentiation and inflammation resolution. Hence, basophils play an essential role in the fidelity of post-MI cardiac repair and the subsequent limitation of long-term adverse cardiac remodeling. CCR2, C-C motif chemokine receptor 2; IL-4R, IL-4 receptor.

immune cells should not be discounted as being less important when delineating the underlying pathophysiology or when designing therapeutic strategies for cardiac repair. Moreover, the Sicklinger et al. results indicate that both basophils and IL-4/IL-13 are important cellular and cytokine components, respectively, of the tissue microenvironment in the infarcted heart that promote reparative macrophage differentiation, adding to the portfolio of factors regulating Ly6 $\mathrm{C}^{\text {lo }}$ macrophage polarization and inflammation resolution in the infarcted heart $(1,2,9)$. Therefore, circumscribed augmentation of basophils and/or IL-4 may represent a fruitful immunomodulatory approach to expanding reparative macrophages and promoting resolution after MI, thereby alleviating the late development of HF. Indeed, consistent with these data, exogenous IL-4 administration acutely after MI has been previously shown to improve healing and remodeling via the modulation of $\mathrm{CD}^{206^{+}}$ macrophages (17).

This important study also raises new questions. Aside from basophils, several other immune cell types in the infarcted heart can produce IL-4 and IL-13 includ- 
ing mast cells, Th2 $\mathrm{CD}^{+} \mathrm{T}$ cells, and iNKT cells. The reasons for the specific importance of basophil-derived IL-4/ IL-13, as opposed to IL-4/IL-13 derived from other cell sources, in the production of the observed myeloid and remodeling responses are unclear. The absence of compensatory IL-4 generation in the hearts of basophil-deficient mice suggests the involvement of other factors, e.g., basophil spatial distribution or as-yet ill-defined cell-cell or cytokine interactions that confer primacy to basophils in regulating the IL-4/IL-13 axis in the infarcted heart. Finally, these studies also raise the interesting question of whether basophils play an important role in the pathogenesis of late remodeling after MI. Specifically, several studies have noted cardiac re-expansion of both innate and adaptive immune cell types in ischemic cardiomyopathy, including increased proliferation and abundance of tissue macrophages in the failing heart (18-20). Moreover, in murine models, the ischemic failing heart is a Th2-predominant chronic inflammatory state (21) with augmented myocardial expression of both IL-4 and IL-13, as well as IL-33, a known migratory and activation factor for basophils. While these conditions collectively support a microenvironment both potentially conducive to and resulting from local expansion of cardiac basophils, further study will be needed to determine whether this is indeed the case, and whether modulation of this rare cell population may also be a viable therapeutic approach in chronic HF.

\section{Conclusions}

Sicklinger et al. (15) have elegantly demonstrated the central importance of cardiac basophils and basophil-derived IL-4 and IL-13 in the inflammatory and healing response after MI. This heretofore unappreciated role for this relatively sparse granulocyte population provides new perspectives on immune cell regulation of post-MI cardiac repair and the ability of these cells to direct the prevailing monocyte/macrophage phenotype in the infarcted heart. Further investigation will be required to delineate the mechanisms underlying the specific effects of basophil-derived IL-4/IL-13 on cardiac macrophages, and the potential role of these cells in the late cardiac remodeling that occurs in ischemic cardiomyopathy and HF.

\section{Acknowledgments}

The author's research program on immune cell activation and HF is supported by NIH R01 grants HL147549 and HL157999.

Address correspondence to: Sumanth D. Prabhu, Professor and Director, Division of Cardiovascular Disease, University of Alabama at Birmingham, 311 Tinsley Harrison Tower, 1900 University Blvd., Birmingham, Alabama 35294-0006, USA. Phone: 205.934.3624; Email: sprabhu@ uab.edu.

1. Prabhu SD, Frangogiannis NG. The biological basis for cardiac repair after myocardial infarction: from inflammation to fibrosis. Circ Res. 2016;119(1):91-112.

2. Hilgendorf I, et al. Ly-6Chigh monocytes depend on Nr4a1 to balance both inflammatory and reparative phases in the infarcted myocardium. Circ Res. 2014;114(10):1611-1622.

3. Kain V, et al. Inflammation revisited: inflammation versus resolution of inflammation following myocardial infarction. Basic Res Cardiol. 2014;109(6):444.

4. Nahrendorf M, et al. The healing myocardium sequentially mobilizes two monocyte subsets with divergent and complementary functions. JExp Med. 2007;204(12):3037-3047.

5. Yan X, et al. Temporal dynamics of cardiac immune cell accumulation following acute myocardial infarction. J Mol Cell Cardiol. 2013;62:24-35.

6. Leuschner F, et al. Therapeutic siRNA silencing in inflammatory monocytes in mice. Nature Biotechnol.2011;29(11):1005-1010.
7. Swirski FK, et al. Identification of splenic reservoir monocytes and their deployment to inflammatory sites. Science. 2009;325(5940):612-616

8. Horckmans M, et al. Neutrophils orchestrate post-myocardial infarction healing by polarizing macrophages towards a reparative phenotype. Eur Heart J. 2017;38(3):187-197.

9. Wan E, et al. Enhanced efferocytosis of apoptotic cardiomyocytes through myeloid-epithelialreproductive tyrosine kinase links acute inflammation resolution to cardiac repair after infarction. Circ Res. 2013;113(8):1004-1012.

10. Anzai A, et al. Regulatory role of dendritic cells in postinfarction healing and left ventricular remodeling. Circulation. 2012;125(10):1234-1245.

11. Hofmann U, et al. Activation of CD4+ T lymphocytes improves wound healing and survival after experimental myocardial infarction in mice. Circulation. 2012;125(13):1652-1663.

12. Weirather J, et al. Foxp3+CD4+ T cells improve healing after myocardial infarction by modulating monocyte/macrophage differentiation. Circ Res. 2014;115(1):55-67.

13. Sobirin MA, et al. Activation of natural killer T cells ameliorates postinfarct cardiac remodeling and failure in mice. Circ Res. 2012;111(8):1037-1047.

14. Zouggari $\mathrm{Y}$, et al. B lymphocytes trigger monocyte mobilization and impair heart function after acute myocardial infarction. Nat Med. 2013;19(10):1273-1280.

15. Sicklinger F, et al. Basophils balance healing after myocardial infarction via IL-4/IL-13. J Clin Invest. 2021;131(13): 136778.

16. Prabhu SD. Cytokine-induced modulation of cardiac function. Circ Res. 2004;95(12):1140-1153.

17. Shiraishi M, et al. Alternatively activated macrophages determine repair of the infarcted adult murine heart. JClin Invest. 2016;126(6):2151-2166

18. Ismahil MA, et al. Remodeling of the mononuclear phagocyte network underlies chronic inflammation and disease progression in heart failure: critical importance of the cardiosplenic axis. Circ Res. 2014;114(2):266-282.

19. Sager HB, et al. Proliferation and recruitment contribute to myocardial macrophage expansion in chronic heart failure. Circ Res. 2016;119(7):853-864.

20. Bajpai G, et al. The human heart contains distinct macrophage subsets with divergent origins and functions. Nat Med. 2018;24(8):1234-1245.

21. Bansal SS, et al. Activated T lymphocytes are essential drivers of pathological remodeling in ischemic heart failure. Circ Heart Fail. 2017;10(3):e003688 